\title{
SUBHARMONIC FUNCTIONS AND UNIFORM ALGEBRAS ${ }^{1}$
}

\author{
DONNA KUMAGAI
}

\begin{abstract}
Recently Aupetit and Wermer [J. Functional Anal. 28 (1978), 386-400] have shown conditions under which analytic structure exists in the spectrum space of a uniform algebra. Their work makes critical use of subharmonicity properties of certain classes of functions. In this paper, we develop a technique which offers an alternate and unified proof for subharmonicity of the functions in their paper assuming Basener's generalized Shilov boundary conjecture. Our technique uses the Oka-Wermer method applied to the $n$-fold tensor product of the algebra. We exhibit further applications of our main result including a special case which holds for all uniform algebras.
\end{abstract}

Introduction. Let $A$ be a uniform algebra on a compact Hausdorff space $X$ with spectrum $M$. We know that in many cases $M \backslash X$ has a natural analytic structure. If the structure is one-dimensional and if $f \in A$, then locally either $f$ is constant or $f$ defines a covering map of a portion of $M \backslash X$ onto a subset of the complex plane. This suggests that the mapping properties of functions in $A$ should be used to exhibit the analytic structure in $M \backslash X$, and indeed some deep results have been obtained along this line [1]. Recently, Aupetit and Wermer proved the following [2], which extended the earlier results by $\mathrm{E}$. Bishop, J. Wermer, and R. F. Basener.

TheOREM 1. Let $W$ be a component of $C \backslash f(X)$. Assume that there exists a subset $G$ of $W$ such that

(1) $G$ has positive exterior capacity.

(2) For each $\lambda \in G$ the fiber over $\lambda, f^{-1}(\lambda)=\{y \in M \mid f(y)=\lambda\}$, is denumerably infinite or finite.

Then there exists an open dense subset of $f^{-1}(W)$ which can be given the structure of one-dimensional complex analytic space such that each $g \in A$ is analytic on this space.

The key tool used in the Aupetit-Wermer paper is the subharmonicity properties of certain classes of functions which arise in the following manner. For $f, g \in A$, suppose $u$ is a set function from compact subsets of $g\left[f^{-1}(W)\right]$ into $\mathbf{R} \cup\{-\infty\}$. Some examples of such functions in the Aupetit-Wermer

Received by the editors April 4, 1978 and, in revised form, July 20, 1978 and January 12, 1979. AMS (MOS) subject classifications (1970). Primary 46J10, 46J15.

Key words and phrases. Subharmonicity, maximal ideal space, generalized Shilov boundary.

'This paper contains the result of the author's Ph.D. thesis written at Lehigh University under the direction of Richard F. Basener. 
paper are: $u_{1}(K)=\log \left\{\max _{z \in K}|z|\right\}, \quad u_{2}(K)=\operatorname{diameter}[K], \quad u_{3}(K)=$ $\log \{$ diameter $[K]\}$. For each such $u_{i}$ associate a function $\mathcal{U}_{i}$ on $W$ by

$$
\mathscr{Q}_{i}(x)=u_{i}\left\{g\left[f^{-1}(x)\right]\right\}, \quad \lambda \in W .
$$

It is the subharmonicity of the $\mathscr{U}_{i}$ that plays a large role in the Aupetit and Wermer approach. The subharmonicity of $\mathscr{U}_{1}$ is established by Wermer in an earlier paper [3] by combining an idea of Oka with results from the theory of uniform algebras; Aupetit and Wermer prove the subharmonicity of $\mho_{2}$ by expressing $u_{2}$ in terms of $u_{1}$; they prove that $\mathscr{U}_{3}$ is subharmonic by applying potential theoretic results and the fact that $\mathscr{\mho}_{2}$ is subharmonic. In all of their proofs the characteristics of the individual functions are utilized in an important way.

In this paper we develop a technique to provide a single proof for the subharmonicity of all the $\mathscr{U}_{i}$ in the Aupetit-Wermer paper and a larger class of functions assuming Basener's generalized Shilov boundary conjecture. A detailed discussion of the conjecture will be given later.

Our technique, modeled in part after Wermer's proof for $\mathscr{Q}_{1}$, is as follows: Consider the tensor product algebra $\bigotimes_{n} A$ of $n$ copies of $A$. Define $\psi$ on a component $W$ of $\Pi_{1}^{n}[f(M) \backslash f(X)]$ by

$$
\begin{aligned}
\psi\left(\lambda_{1}, \ldots, \lambda_{n}\right)=\log \left\{\max \left|G\left(\phi_{1}, \ldots, \phi_{n}\right)\right| ;\left(\phi_{1}, \ldots, \phi_{n}\right)\right. & \\
& \left.\in(f, \ldots, f)^{-1}\left(\lambda_{1}, \ldots, \lambda_{n}\right)\right\}
\end{aligned}
$$

where $G \in \otimes_{n} A$. Using the Oka-Wermer method we show in Theorem 2 that $\psi$ is plurisubharmonic.

Corollary 1 shows that if $n=2$ then the restriction of $\psi$ on the diagonal of $W$ establishes the subharmonicity of all $\mathscr{U}_{i}$ 's in the Aupetit-Wermer paper.

Corollary 2 shows that functions associated with transfinite diameter and capacity are subharmonic.

The generalized Shilov boundary condition, which Aupetit and Wermer did not require, is required in our proof because we have put their problem into the context of higher dimensional algebras and functions of several complex variables. It has been conjectured that this hypothesis can be removed, and recently R. F. Basener has verified that the generalized Shilov boundary condition holds for a large class of uniform algebras ([4], [5]). Our hypothesis is independent of conditions (1) and (2) in the Aupetit-Wermer theorem. Thus, our approach offers a potentially comprehensive and efficient technique for proving subharmonicity of functions associated with a uniform algebra.

The tensor product $\otimes_{i=1}^{n} A_{i}$ of uniform algebras $A_{i}$ on $X_{i}$ is the smallest closed subalgebra of $C\left(\Pi_{i=1}^{n} X_{i}\right)$ generated by functions of the form $h_{1}\left(x_{1}\right) \cdots h_{n}\left(x_{n}\right)$, where $h_{i} \in A_{i}$ and $x_{i} \in X_{i}$. By $\otimes_{n} A$ we mean the tensor product of $n$ copies of $A$.

Let $A^{n}=\left\{\left(f_{1}, \ldots, f_{n}\right) \mid f_{1}, \ldots, f_{n} \in A\right\}$, so that each $F=\left(f_{1}, \ldots, f_{n}\right) \in$ $A_{n}$ maps $M$ into $\mathbf{C}^{n}$. If $K$ is a compact subset of $M$ let $A_{K}=\{f \in C(K) \mid f$ is a 
uniform limit on $K$ of functions from $A\}$. For $F \in A^{n}$, let $V(F)=\{x \in$ $M \mid F(x)=0\} . V(F)$ is $A$-convex, i.e. the maximal ideal space of $A_{V(F)}$ is $V(F)$.

Definition. $\partial_{n} A=\operatorname{cl}\left[\cup\left\{\partial_{0} A_{V(F)} \mid F \in A^{n}\right\}\right] . \partial_{n} A$ is called the $n$th Shilov boundary of $A . \partial_{0} A$ is the usual Shilov boundary of $A$. It has been conjectured that the following condition is true for arbitrary uniform algebras, $A_{j}$, $j=1, \ldots, n$ :

$$
\partial_{i}\left[\bigotimes_{j=1}^{n} A_{j}\right]=\bigcup_{i_{1}+\cdots+i_{j}=i} \prod_{j=1}^{n} \partial_{i_{j}} A_{j}
$$

R. F. Basener [5] has recently proved the condition to be true for $\bigotimes_{j=1}^{n} A_{j}$ where $A_{j}=P\left(X_{j}\right)$ and $X_{j}$ is polynomial polyhedron. He has also shown that if $A_{1}$ is an arbitrary uniform algebra and $A_{2}$, any polydisc algebra, then $A_{1} \otimes A_{2}$ satisfies (*) for $i=0,1$. We are ready for our main theorem.

Theorem 2. Let $X, A$, and $M$ be as before. Assume $\otimes_{n} A$ satisfies the condition (*). For $f \in A$ let $\mathcal{F}$ be the map on $\Pi_{1}^{n} M$ with $\mathscr{F}\left(\varphi_{1}, \ldots, \varphi_{n}\right)=$ $\left(f\left(\varphi_{1}\right), \ldots, f\left(\varphi_{n}\right)\right)$. Let $W$ be a component of $\Pi_{1}^{n}[f(M) \backslash f(X)]$. For $G \in \Theta_{n} A$ and $\lambda \in W$,put

$$
\psi(\lambda)=\log \left\{\max |G(\varphi)|: \varphi \in \mathscr{F}^{-1}(\lambda)\right\} .
$$

Then $\psi$ is plurisubharmonic on $W$.

Proof. First we show $\psi$ is upper semicontinuous on $W$. Fix $\lambda \in W$. Assume $\left\{\lambda^{k}\right\}_{k=1}^{\infty} \rightarrow \lambda$. Suppose $\psi\left(\lambda^{k}\right) \geqslant l>-\infty$ for all $k$. We claim $\psi(\lambda) \geqslant l$ :

$$
\psi\left(\lambda^{k}\right)=\max \left\{\log \left|G\left(\varphi^{k}\right)\right|: \varphi^{k} \in \mathcal{F}^{-1}\left(\lambda^{k}\right)\right\} .
$$

Since $\mathscr{F}^{-1}\left(\lambda^{k}\right)$ is compact for each $k$, there exists $\varphi^{k} \in \Pi_{1}^{n} M$, the maximal ideal space of $\otimes_{n} A$, such that $\varphi^{k}=\mathscr{F}^{-1}\left(\lambda^{k}\right)$ and $\log \left|G\left(\varphi^{k}\right)\right| \geqslant l$. By compactness of $\Pi_{1}^{n} M$ we can find a subnet $\left\{\varphi^{\alpha}\right\}$ of $\left\{\varphi^{k}\right\}$ which converges to $\varphi$ in $\Pi_{1}^{n} M$.

$$
\begin{gathered}
\mathscr{F}(\varphi)=\lim _{\alpha} \mathscr{F}\left(\varphi^{\alpha}\right)=\lim _{k} \mathscr{F}\left(\varphi^{k}\right)=\lim _{k} \lambda^{k}=\lambda, \\
\log |G(\varphi)|=\lim _{\alpha} \log \left|G\left(\varphi^{\alpha}\right)\right| \geqslant l .
\end{gathered}
$$

So, $\psi(\lambda)=\max \left\{\log |G(\varphi)|: \varphi \in \mathscr{F}^{-1}(\lambda)\right\}>l$. This shows that $\psi$ is u.s.c. on $W$.

It remains to be shown that if $T$ is an arbitrary linear transformation, $\psi \circ T$ is subharmonic on $W$ in each variable. We will show $\psi$ restricted to a given complex line, $L$, in $W$ is subharmonic. Let

$$
L=\bigcap_{j=1}^{n-1}\left\{\left(\lambda_{1}, \ldots, \lambda_{n}\right) \in W \mid \sum_{i=1}^{n} \alpha_{i j} \lambda_{i}=\gamma_{j}\right\} ; \quad \alpha_{i j}, \gamma_{j} \in \mathbf{C} .
$$

Let $\Delta$ be a closed disc contained in $L$ and $\partial \Delta$ be the boundary of $\Delta$ in the topology of $L$. Choose a polynomial $P$ such that $\left.\psi\right|_{L} \leqslant \operatorname{Re} P$ on $\partial \Delta$; i.e., $\left.\psi\right|_{L} \cdot\left|e^{-P}\right|<1$ on $\partial \Delta$. 


$$
\begin{aligned}
& \text { Put } f_{i}\left(\varphi_{1}, \ldots, \varphi_{n}\right)=f\left(\varphi_{i}\right) ; \\
& \begin{aligned}
F^{j} & =\sum_{i=1}^{n} \alpha_{i j} f_{i}-\gamma_{j}, \quad j=1, \ldots, n-1 ; \\
V & =\left\{\varphi \in \prod_{1}^{n} M \mid F^{j}(\varphi)=0, j=1, \ldots, n-1\right\} ; \\
\tilde{X} & =f^{-1}[f(X)] .
\end{aligned}
\end{aligned}
$$

Let $Q_{i}$ be the Cartesian product of $\tilde{X}$ with $n-1$ copies of $M$ in which $\tilde{X}$ appears at the $i$ th entry. Thus, $Q_{1}=\tilde{X} \times M \times \cdots \times M$. From the hypothesis we have,

$$
\partial_{n-1} \bigotimes_{n} A=\bigcup_{\substack{j_{1}+\cdots+j_{n}=n-1 ; \\ j_{1} j_{2}, \ldots, j_{n}>0}}\left[\partial_{j_{1}} A \times \partial_{j_{2}} A \times \cdots \times \partial_{j_{n}} A\right] \subseteq \bigcup_{i=1}^{n} Q_{i} .
$$

Let $\otimes_{n} A_{V}$ be the restriction algebra, $\{g \in C(V) \mid g$ is a uniform limit on $V$ of functions from $\otimes_{n} A$ \}. Let $\partial_{0} \otimes_{n} A_{V}$ to be the usual Shilov boundary of $\otimes_{n} A_{V}$. By the definition of generalized Shilov boundary, it follows that, $\partial_{0} \otimes_{n} A_{V} \subseteq \partial_{n-1} \otimes_{n} A \subseteq \cup{ }_{i=1}^{n} Q_{i}$.

Denote $N=\mathscr{F}^{-1}(\Delta)$. We claim $N \subseteq V \backslash \partial_{0} \otimes_{n} A_{V}$. To prove the claim, we

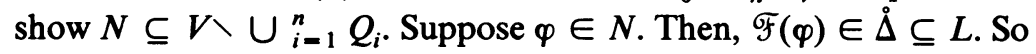

$$
\sum_{i=1}^{n} \alpha_{i j} f_{i}(\varphi)=\gamma_{j}, \quad j=1, \ldots, n-1
$$

Hence, $\varphi \in V$. If $\varphi \in \cup_{i=1}^{n} Q_{i}$ then $\varphi_{i} \in \tilde{X}$ for some $1 \leqslant i \leqslant n$. But, $f\left(\varphi_{i}\right) \in$ $f(M) \backslash f(X)$. So $\varphi_{i} \notin \tilde{X}$. Hence $N \subset V \backslash \cup{ }_{i=1}^{n} Q_{i}$ as claimed.

Fix $\xi \in \stackrel{\Delta}{ }$ and choose $\theta=\left(\theta_{1}, \ldots, \theta_{n}\right) \in \mathscr{F}^{-1}(\xi)$ on which $|G|$ takes the maximum. By the local maximum modulus principle of $\otimes_{n} A_{V}$ applied to $N$,

$$
|G(\theta)| \cdot\left|e^{-P\left(f\left(\theta_{1}\right), \ldots, f\left(\theta_{n}\right)\right)}\right| \leqslant|G(\eta)| \cdot\left|e^{-P\left(f\left(\eta_{1}\right), \ldots, f\left(\eta_{n}\right)\right)}\right|
$$

for some $\eta \in \partial N$, where $\partial N$ is the boundary of $N$ relative to $V$. But, $\partial N \subseteq \mathscr{F}^{-1}(\partial \Delta)$. So

$$
|G(\theta)| \cdot\left|e^{-P\left(f\left(\theta_{1}\right), \ldots, f\left(\theta_{n}\right)\right)}\right| \leqslant 1 .
$$

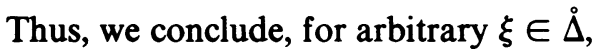

$$
\log \max \left\{|G(\varphi)|: \varphi \in \mathscr{F}^{-1}(\xi)\right\} \leqslant \operatorname{Re} P(\xi) .
$$

We have shown that $\psi$ is plurisubharmonic on $W$ under the hypothesis (*); however, we show in Theorem 3 that $\psi$ is $n$-subharmonic without any hypothesis on the generalized Shilov boundary.

Recall that an upper semicontinuous function $\psi$ on an open subset $\Omega$ of $\mathbf{C}^{n}$ is $n$-subharmonic if $-\infty \leqslant \psi<\infty, \psi \neq-\infty$ on any component of $\Omega$ and $\psi$ is subharmonic in each variable. 
LEMMA 1. Suppose $u$ is upper semicontinuous on an open subset $\Omega$ of $\mathbf{C}^{n}$, and $-\infty<u<\infty$. Assume $u \neq-\infty$ on any component of $\Omega$. If $u$ satisfies the condition below, then $u$ is $n$-subharmonic:

Given any polydisc $D \subseteq \Omega$ and a polynomial $P$ on $C^{n}$, if $u<\operatorname{Re} P$ on $\partial^{0} D$, the distinguished boundary of $D$, then $u \leqslant \operatorname{Re} P$ on $D$.

THEOREM 3. Notations as before.

$$
\psi(\lambda)=\log \left\{\max |G(\theta)| ; \theta \in \mathcal{F}^{-1}(\lambda)\right\}
$$

is $n$-subharmonic on $W$.

Proof. For simplicity of notation we prove the theorem for $n=2$. The same argument holds for the general case. We have shown in Theorem 2 that $\psi$ is u.s.c. on $W$. It suffices to show that $\psi$ satisfies the condition of Lemma 1 .

Let $D=D_{1} \times D_{2}$ be a bidisc in $W$, with $\partial^{0} D$ its distinguished boundary. Choose a polynomial $P$ such that $\psi \leqslant \operatorname{Re} P$ on $\partial^{0} D$. That is, suppose for each $\left(y_{1}, y_{2}\right) \in \partial^{0} D$, if $\left(\varphi_{1}, \varphi_{2}\right) \in \mathscr{F}^{-1}\left(y_{1}, y_{2}\right)$. Then

$$
\left|G\left(\varphi_{1}, \varphi_{2}\right)\right| \cdot\left|e^{-P\left(f\left(\varphi_{1}\right), f\left(\varphi_{2}\right)\right)}\right|<1 .
$$

Let $\left(x_{1}, x_{2}\right)$ be an arbitrary point in $D^{\circ}$. By compactness of $\mathscr{F}^{-1}\left(x_{1}, x_{2}\right)$, there is a point $\left(\theta_{1}, \theta_{2}\right) \in \mathcal{F}^{-1}\left(x_{1}, x_{2}\right)$ on which $|G|$ takes the maximum value. We will show

$$
\left|G\left(\theta_{1}, \theta_{2}\right)\right| \cdot\left|e^{-P\left(x_{1}, x_{2}\right)}\right| \leqslant 1 .
$$

Fix $\theta_{1}$. Define $H(\theta)=G\left(\theta_{1}, \theta\right) \cdot e^{-P\left(f\left(\theta_{1}\right), f(\theta)\right)}$ for $\theta \in M$. It is easy to show that $H \in A$. Recall that $\left(\theta_{1}, \theta_{2}\right) \in \mathscr{F}^{-1}\left(x_{1}, x_{2}\right)$ where $\left(x_{1}, x_{2}\right) \in D^{\circ}$. Hence, $\theta_{2} \in f^{-1}\left(\dot{D}_{2}\right)$. Applying the local maximum modulus principle for $A$ to $f^{-1}\left(\check{D}_{2}\right)$, we obtain $\left|H\left(\theta_{2}\right)\right| \leqslant\left|H\left(\theta_{2}^{\prime}\right)\right|$ for some $\theta_{2}^{\prime} \in \partial\left[f^{-1}\left(\check{D}_{2}\right)\right]$. Note $\partial_{0} A \cap$ $f^{-1}\left(D_{2}\right)=\varnothing$. We have,

$$
\left|G\left(\theta_{1}, \theta_{2}\right)\right| \cdot\left|e^{-P\left(f\left(\theta_{1}\right), f\left(\theta_{2}\right)\right)}\right| \leqslant\left|G\left(\theta_{1}, \theta_{2}^{\prime}\right)\right| \cdot\left|e^{-P\left(f\left(\theta_{1}\right) f\left(\theta_{2}^{\prime}\right)\right)}\right| .
$$

Now fix $\theta_{2}^{\prime}$. Let $V=\left\{\left(\theta, \theta_{2}^{\prime}\right) \mid \theta \in f^{-1}\left(D_{1}\right)\right\}$. We claim that $\partial_{0}\left(A \otimes A_{V}\right) \subset$ $\mathcal{F}^{-1}\left(\partial^{0} D\right)$.

Proof of THE Claim. For each $h \in A \otimes A$, define $\tilde{h}(x)=h\left(x, \theta_{2}^{\prime}\right)$. Then $\tilde{h} \in A$. Apply the local maximum modulus principle to $f^{-1}\left(\dot{D}_{1}\right)$ and obtain

$$
\|\tilde{h}\| \overline{f^{-1}\left(\dot{D}_{1}\right)}=\|\tilde{h}\|_{\partial}\left[\overline{f^{-1}\left(\dot{D}_{1}\right)}\right]
$$

So, for each $h \in A \otimes A$ and $\left(x, \theta_{2}^{\prime}\right) \in V$, we can find some

$$
\left(x^{\prime}, \theta_{2}^{\prime}\right) \in \partial\left[f^{-1}\left(\stackrel{D}{1}_{1}\right)\right] \times f^{-1}\left(\partial D_{2}\right) \subseteq f^{-1}\left(\partial D_{1}\right) \times f^{-1}\left(\partial D_{2}\right)=\mathscr{F}^{-1}\left(\partial^{0} D\right)
$$

such that $\left|h\left(x, \theta_{2}^{\prime}\right)\right| \leqslant\left|h\left(x^{\prime}, \theta_{2}^{\prime}\right)\right|$. This shows that $\mathscr{F}^{-1}\left(\partial^{0} D\right) \cap V$ is a closed boundary for $A \otimes A_{V}$. Hence $\partial_{0}\left(A \otimes A_{V}\right) \subseteq \mathscr{F}^{-1}\left(\partial^{0} D\right)$. Hence there exists $\left(\xi, \theta_{2}^{\prime}\right) \in \partial_{0}\left(A \otimes A_{V}\right)$ such that

$$
\left|G\left(\theta_{1}, \theta_{2}^{\prime}\right)\right| \cdot\left|e^{-P\left(f\left(\theta_{1}\right) f\left(\theta_{2}^{\prime}\right)\right)}\right| \leqslant\left|G\left(\xi, \theta_{2}^{\prime}\right)\right| \cdot\left|e^{-P\left(f(\xi) f\left(\theta_{2}^{\prime}\right)\right)}\right| .
$$


By (2), (3), and (1),

$$
\left|G\left(\theta_{1}, \theta_{2}\right)\right| \cdot\left|e^{-P\left(x_{1}, x_{2}\right)}\right| \leqslant\left|G\left(\xi, \theta_{2}^{\prime}\right)\right| \cdot\left|e^{-P\left(y_{1} y_{2}\right)}\right| \leqslant 1 .
$$

This concludes the proof of Theorem 3.

In the remainder of this paper, we shall discuss applications of Theorem 2. In [2], B. Aupetit and J. Wermer developed sufficient conditions for the existence of analytic structure in the maximal ideal space of a uniform algebra, improving on the earlier results by R. Basener [6] and E. Bishop [7]. Aupetit and Wermer's proof depends critically on subharmonicity properties exhibited by certain classes of functions associated with the uniform algebra. Presently, we shall focus our attention on these functions.

Notation. Fix $g \in A$. For $\lambda \in W$, a component of $\mathbf{C} \backslash f(X)$, denote:

$$
\begin{aligned}
K_{g}(\lambda) & =g\left[f^{-1}(\lambda)\right]=\left\{g(y): y \in f^{-1}(\lambda)\right\} \subset \mathbf{C} \\
Z_{g}(\lambda) & =\max \left\{|Z|: Z \in K_{g}(\lambda)\right\} \\
r_{g}(\lambda) & =\max \left\{1 /|Z|: Z \in K_{g}(\lambda)\right\} \\
d_{g}(\lambda) & =\text { diameter of } K_{g}(\lambda) .
\end{aligned}
$$

Aupetit and Wermer constructed the following functions and have shown that each of them is subharmonic on $W$ for all $f$ and $g$ in $A$ :

Function 1: $\lambda \rightarrow Z_{g}(\lambda)$;

Function 2: $\lambda \rightarrow \log Z_{g}(\lambda)$;

Function 3: $\lambda \rightarrow d_{g}(\lambda)$;

Function 4: $\lambda \rightarrow|\exp P(x)| \cdot d_{g}(\lambda)$, where $P$ is a polynomial;

Function 5: $\lambda \rightarrow \log d_{g}(x)$.

They have also shown that for fixed $\lambda_{0} \in W$, and a complex number $\alpha$ with $\alpha \notin \hat{K}_{g}\left(\lambda_{0}\right)$, the polynomial hull of $K_{g}\left(\lambda_{0}\right)$,

Function 6: $\lambda \rightarrow r_{g-\alpha}(\lambda)$ is subharmonic in a neighborhood of $\lambda_{0}$.

Subharmonicity of certain classes of functions associated with $K_{g}(\lambda)$ including the ones listed above follows from Theorem 2.

Corollary 1 to Theorem 2. Suppose $A \otimes A$ satisfies the hypothesis (*). Then Functions (1)-(6) listed above are subharmonic.

Proof. If we let $n=2$, Theorem 2 states that

$$
\psi\left(\lambda_{1}, \lambda_{2}\right)=\log \max \left\{\left|G\left(\theta_{1}, \theta_{2}\right)\right| \mid\left(\theta_{1}, \theta_{2}\right) \in \mathscr{F}^{-1}\left(\lambda_{1}, \lambda_{2}\right)\right\}
$$

is plurisubharmonic. Hence $\psi$ is subharmonic in each variable. Let $G\left(\theta_{1}, \theta_{2}\right)$ $=g\left(\theta_{1}\right)$. Then $\log \max \left\{\left|g\left(\theta_{1}\right)\right|: \theta_{1} \in f^{-1}(\lambda)\right\}$ is subharmonic. This shows that Function 2 is subharmonic. If $\log |h|$ is subharmonic so is $|h|$. Hence Function 1 is subharmonic. $\psi$ is subharmonic on the diagonal of each component of $W$. By setting $G\left(\theta_{1}, \theta_{2}\right)=g\left(\theta_{1}\right)-g\left(\theta_{2}\right)$ we have, $\log \max \left\{\mid g\left(\theta_{1}\right)-\right.$ $\left.g\left(\theta_{2}\right) \mid:\left(\theta_{1}, \theta_{2}\right) \in \mathscr{F}^{-1}(\lambda, \lambda)\right\}$ is subharmonic. This shows that Functions 3 and 5 are subharmonic. $\log \left(|\exp P(\lambda)| \cdot d_{g}(\lambda)\right)=\operatorname{Re} P(\lambda)+\log d_{g}(\lambda)$. So, Function 4 is subharmonic. Subharmonicity of Function 6 is proved in [2] via subharmonicity of $\log Z_{g}$, Function 2 . 
Corollary 2 to Theorem 2. Suppose $\bigotimes_{n} A$ satisfies the condition (*). Then $\lambda \rightarrow$ Capacity $\left[K_{g}(\lambda)\right]$ is subharmonic on each component of $f(M) \backslash f(X)$.

A theorem by Szegö relates capacity to a geometric quantity called transfinite diameter [8]. Diameter of order $n$ of a closed bounded set, $E$, denoted by $d_{n}(E)$ is

$$
d_{n}(E)=\max \left\{\Pi\left|z_{i}-z_{j}\right|^{2 / n(n-1)}, z_{i}, z_{j} \in E, 1 \leqslant i<j<n\right\} .
$$

If $n=2, d_{n}(E)$ is the ordinary diameter of $E$. It is straightforward to show that $d_{n}<d_{n-1} \leqslant \cdots \leqslant$ diameter $(E)<\infty$. We set $d=\lim _{n} d_{n}$ and call it the transfinite diameter.

Theorem 4 (Szegö). The capacity of a closed bounded set is equal to its transfinite diameter.

We are now ready to prove Corollary 2.

Proof. Since the monotone decreasing limit of subharmonic functions is subharmonic, we need only to prove that for all $n \geqslant 2, \lambda \rightarrow d_{n}\left[K_{g}(\lambda)\right]$ is subharmonic. We shall use the notation $d_{n}(\lambda)$ to mean $d_{n}\left[K_{g}(\lambda)\right]$. If $g \in A$ and $\varphi=\left(\varphi_{1}, \ldots, \varphi_{n}\right) \in \Pi_{1}^{n} M$, denote $g_{i}(\varphi)=g\left(\varphi_{i}\right), i=1, \ldots, n . g_{i} \in \bigotimes_{n} A$ for each $i$. Hence, $\Pi_{1<i<j \leqslant n}\left(g_{i}-g_{j}\right) \in \otimes_{n} A$. For $\left(\lambda_{1}, \ldots, \lambda_{n}\right) \in$ $\Pi_{1}^{n}[f(M) \backslash f(X)]$ put

$$
V_{n}\left(\lambda_{1}, \ldots, \lambda_{n}\right)=\max \left\{\prod_{1<i<j<n}\left|g_{i}(\varphi)-g_{j}(\varphi)\right|: \varphi \in \mathscr{F}^{-1}\left(\lambda_{1}, \ldots, \lambda_{n}\right)\right\} .
$$

Then $d_{n}(\lambda)=V_{n}(\lambda, \ldots, \lambda)^{2 / n(n-2)}$, i.e., $d_{n}$ is $V_{n}^{2 / n(n-2)}$ restricted to the diagonal. By Theorem $2, \log V_{n}$ is plurisubharmonic on $W$, hence subharmonic on the diagonal of $W$. This establishes subharmonicity of $d_{n}$, since

$$
\log d_{n}=\frac{2}{n(n-1)} \log V_{n} \text { and } \frac{2}{n(n-1)}>0 \text {. }
$$

\section{BIBLIOGRAPHY}

1. J. Wermer, Banach algebras and several complex variables, Springer-Verlag, Berlin and New York, 1976, pp. 64-70, pp. 137-142.

2. B. Aupetit and J. Wermer, Capacity and uniform algebras, J. Functional Anal. 28 (1978), 386-400.

3. J. Wermer, Subharmonicity and hulls, Pacific J. Math. 8 (1975), 283-290.

4. R. F. Basener, A generalized Shilov boundary and analytic structure, Proc. Amer. Math. Soc. 47 (1975), 98-104.

5. Boundaries for product algebras (preprint).

6. _ A condition for analytic structure, Proc. Amer. Math. Soc. 36 (1972), 156-160.

7. E. Bishop, Holomorphic completions, analytic continuation, and the interpolation of seminorms, Ann. of Math. (2) 78 (1963), 468-500.

8. G. M. Goluzin, Geometric theory of functions of a complex variable, Transl. Math. Monographs, vol. 26, Amer. Math. Soc., Providence, R. I., 1969.

Department of Mathematics, Lehigh University, Bethlehem, Pennsylvania 18015

Current address: Department of Mathematics, Pennsylvania State University, Hazleton, Pennsylvania 18201 